\title{
Ueber den Einfluss einiger Salze und Alkaloiden auf die Verdauung.
}

\author{
Von \\ Dr. Lonis Wolberg.
}

Von der Warschauer medic. Facultät gekrönte Preisschrift' $\left.{ }^{1}\right)$.

Es ist eine allgemein bekannte Thatsache, dass durch Kochsalz die Fäulniss gehemmt wird, das ist, Kochsalz besitzt die Eigenschaft, die Fermentbildung, von der die Fäulniss im Allgemeinen abhängig ist, zu hemmen. Auch andere Salze besitzen dieselbe Eigenschaft; so werden z. B. die Lösungen von $\mathrm{MgSO}_{4}$ und $\mathrm{Na}_{2} \mathrm{SO}_{4}$ in America und England zur Aufbewahrung anatomischer Präparate benutzt und vertreten dort die Stelle des bei uns an diesen Zwecken gebräuchlichen Alcohols. Diese Thatsachen waren Allen seither bekannt; unlängst aber fing auch die Wissenschaft sich mit dieser wichtigen Frage zu beschäftigen an; wichtig ist die Frage deshalb, weil die Salze nicht nur in der Diätetik, sondern auch in der Therapie eine grosse Rolle spielen; ausserdem die uns bekannte Thatsache, dass durch einige Salze die Fäulniss gehemmt wird, führt uns in logischer Gedankenreihe zu folgenden Fragen: 1) Welche Salze hemmen die Fermentation? 2) Bei wie grosser Menge hemmt das betreffende Salz? 3) Wirkt ein und dasselbe Salz auf eine und dieselbe Weise auf verschiedene Fermentationen? 4) Sind Salze vorhanden, durch welche die Fermentation nicht gehemmt, sondern beschleunigt wird? 5) Durch welchen Bestandtheil des Salzes wird die Fermentation beeinflusst, durch die Basis, oder durch die Säure? etc. etc. Die Frage weiter dehnend, können wir neugierig sein, wie die so oft gebrauchten Alealoide auf die Fermentation wirken!

Trotz der Wichtigkeit dieser Fragen, wurden sie erst in

1) Verkürzt und übersetzt vom Verfasser. 
den letzten Jahren ventilirt; Liebig ${ }^{1}$ ) eröffnete die Reihe indem er sagte, dass die Fermentationskraft der Hefe durch eine Zugabe von $\mathrm{NaCl}$ oder $\mathrm{KCl}$ ein wenig verstärkt wird. Seitdem arbeiteten an diesem Thema: Heidenhain ${ }^{2}$ ), Wilhelm Ebstein und Julius Muller ${ }^{3}$ ), Carl Knapp ${ }^{4}$ ), A lex.Schmidt ${ }^{5}$ ) und ganz besonders Otto Nasse ${ }^{6}$ ). Alle diese Autoren veranstalteten Experimente iiber den Einfluss der Salze (auch der Alkaloide) auf die Fermente der Bauchspeicheldriise, der Leber, des Mundspeichels; die Magenfermentation aber, unbegreiflicher Weise, blieb nuberïcksichtigt bis jetzt und deshalb wählte ich dieselbe zum Thema meiner Untersuchungen, deren Resultate ich den geneigten Lesern hiemit vorlege. Meine Experimente machte ich im Jahre 1878 7). Ich will nur die wichtigsten Schlïsse aus den fritheren Arbeiten anführen, damit der Leser sie später mit den mẹinigen vergleichen kann. So sagt Carl Knapp: 1) Die Natronsalze hemmen die Fermentation (Alkoholgährung) mit Ausnahme des $\mathrm{Na}_{2} \mathrm{SO}_{4}$, das dieselbe beschleunigt. Da nun aus meinen Experimenten klar wird, dass die Magenfermentation durch $\mathrm{Na}_{2} \mathrm{SO}_{4}$ ebenfalls stark gehemmt wird, so komme ich zur Folgerung, dass ein und dasselbe Salz auf zweierlei Fermente verschieden wirken kann. 2) Die Kalisalze beschleunigen die Fermentation, aber nur auf kurze Zeit; bei längerem Einwirken dieser Salze wird die Beschleunigung der Fermentation immer geringer, folglich, nach Knapp's Erklärung, wird die Hefen-Zelle Anfangs durch die Kalisalze gereizt und deshalb ihre Wirkungsfähigkeit vergrössert, später jedoch werden die Kalisalze indifferent, so dass wir weder Beschleunigung, noch Hemmung des Fermentationsprozesses bemerken.

1) Ueber Gährung, Quelle der Muskelkraft und Ermährung. Separatabdruck aus den Annalen der Chemie und Pharmacie. 1870. S. 61.

2) Beiträge zur Kenntniss des Pankreas (Pflüger's Archiv Bd. X.).

3) Ueber den Einfluss der Säuren und Alkalien auf das Leberferment (Berichte d. deutschen chemischen Gesellschaft S. 679).

4) Ueber den Einfluss der Kali- und Natronsalze auf die Alkoholgährung. Annalen der Chemie und Pharmacie. Seite 65.

5) Ueber die Beziehung des Kochsalzes zu einigen thierischen Fermentationsprozessen (Pflüger's Archiv. Bd. XIII. S. 951).

6) Untersuchungen über die ungeformten Fermente (ibidem. Bd. XI. S. 138).

7) Im Warschauer Institut für physiologische Chemie (Director Dr. H. Fudakowski, bereits verstorben). 
Der Arbeit Wilbelm Ebstein's und Julius Müller's (siehe oben 1. c.) entnehmen wir folgende Sätze: 1) Alkalien hemmen die Fermentation. 2) Durch Säuren wird die Fermentation total gehemmt, aber nicht aufgehoben, weil, nachdem man die sauren Lösungen wieder alkaliseh gemacht, die Fermentation sich wieder einfindet. 3) Diejenigen Salze, die Krystallwasser enthalten, hemmen die Fermentation geringer, als die durch Schmelzen von Wasser befreiten Salze, folglich können wir behaupten, dass die Wirkung der Salze auf Fermente darin besteht, dass sie denselben das Wasser entziehen. Auch meine Experimente (s. unten Tab. III, IV, XII) mit geschmolzenem und crystallischen $\mathrm{Na}_{2} \mathrm{SO}_{4}$ und $\mathrm{Na}_{2} \mathrm{~B}_{4} \mathrm{O}_{7}$ haben zur Genüge die Wahrheit der dritten Bemerkung Ebstein's und Müller's nachgewiesen.

Alexander Schmidt (l. c.) beweist, dass $\mathrm{NaCl}$ die Milchgerinnung hemmt; die neutralen Salze der Alkalien hemmen die Verdatung, weil sie das Aufquellen des Faserstoffs in Flüssigkeit aufhalten. (Es war mir vergönnt auch dies bei grossen Mengen verschiedener Salze zu bemerken.) Weiter sagt Schmidt, dass die Fibringerinnung durch Zugabe kleiner Quantitäten Kochsalz bis zu einer gewissen Grenze gesteigert, später aber, bei grösseren Quantitäten gehemmt wird, um bei noch grösseren Mengen ganz anszubleiben d. i. dass Blut bleibt flüssig. Die grösste Beschleunigung sah Schmidt bei $0,4-0,1 \%$ Kochsalz; bei $2-2,5 \%$ war schon die Hemmung bemerkbar, bei noch grösseren Mengen blieb das Blut fliussig. Weiter beweist Schmidt, dass die Fermentwirkung am stärksten durch $\mathrm{MgSO}_{4}$, dann durch $\mathrm{NaCl}$, am schwächsten aber durch $\mathrm{Na}_{2} \mathrm{SO}_{4}$ gehemmt wird. Warum die Fermentation durch geringe Salzmengen beschleunigt, durch grösșere aber gehemmt wird, ist vorläufig von Schmidt und Anderen unbeantwortet geblieben.

Otto Nasse's zahlreiche Versuche beweisen folgendes: Das Sauerwerden der Muskelsubstanz wird am stärksten durch $\mathrm{NaNO}_{3}$ gehemmt; ihm folgen $\mathrm{KCl}, \mathrm{NaCl}$ und zuletzt $\mathrm{KNO}_{3}$. Die Zuckerinversion (Rohrzucker und Invert-Ferment der Hefe) wird durch $\mathrm{Na}_{2} \mathrm{SO}_{4}$ ein wenig beschleunigt, viel beträchtlicher aber durch $\left(\mathrm{NH}_{4}\right)_{2} \mathrm{SO}_{4}$; eine Hemmung sah $\mathrm{Nasse}$ bei $\mathrm{K}_{2} \mathrm{SO}_{4}$. Diese Resultate stimmten mit denen, die $\mathrm{Nasse}$ früher ïber die Inversion des Zuckers unter dem Einflusse der Schwefelsäure in Anwesenheit schwefelsaurer Salze veranstaltete, nicht ïberein, da 
er in jenen Versuchen (l. c. S. 148. Versuch III u. IV) bei allen schwefelsaurenSalzen eine sehr beträchtliche Hemmung der Zucker-Inversion erhielt. Anf diese Weise bewies Nasse den oben mitgetheilten Satz Carl Knapp's (Seite 5). $\mathrm{NaCl}$ und $\mathrm{KCl}$ hemmen die Inversion, $\mathrm{NH}_{4} \mathrm{Cl}$ beschleunigt sie sehr stark; aber diese Beschleunigung steht im umgekehrten Verhältniss zur Menge des $\mathrm{NH}_{4} \mathrm{Cl}$, so z. B. bei $4 \%$ Salzgehalt ist die Beschleunigung $64 \%$ gleich, bei $16 \%$ hingegen ist sie Null. Von salpetersauren Salzen wird die Inversion durch $\mathrm{NaNO}_{3}$ ein wenig, durch $\mathrm{KNO}_{3}$ etwas mehr gehemmt, durch $\mathrm{NH}_{4} \mathrm{NO}_{3}$ aber sehr stark gefördert, so dass bei $16 \%$ Salz die Beschleunigung immer noch $24 \%$ beträgt, bei $4 \%$ Salzgehalt ist sie $75 \%$ gleich. In den Natrium-Verbindungen hemmt die $\mathrm{HNO}_{3}$ stärker als die $\mathrm{HCl}$; die $\mathrm{H}_{2} \mathrm{SO}_{4}$ hingegen befördert; in den Kalisalzen hemmt $\mathrm{HCl}$ stärker als $\mathrm{HNO}_{3}$, und $\mathrm{H}_{2} \mathrm{SO}_{4}$ beschleunigt abermals. Bei allen Ammoniak-Salzen ist eine Beschleunigung der Inversion sichtbar, die jedoch bei schwefelsaurem Ammon grösser ist, als bei den andern.

Da ich in meinen Versuchen keine Beschleunigung, wohl aber eine Hemmung der Verdaung durch $\mathrm{H}_{2} \mathrm{SO}_{4}$ gesehen habe, so folgere ich daraus, im Vergleich mit Nasse's Resultaten, das nicht nur Salze, sondern auch Säuren auf verschiedene Fermente einen verschiedenen Einfluss üben.

Weiter sieht Nasse, dass bei Speichel und Pankreasferment die Gährung durch $\mathrm{Na}_{2} \mathrm{SO}_{4}, \mathrm{NaCl}, \mathrm{KNO}_{3}$ beschleunigt, bei Diastase aber gehemmt wird. $\mathrm{NH}_{4} \mathrm{NO}_{3}$ hemmt sehr stark bei Speichel und Diastase, beschleunigt bei Pankreas u. s. w. Nasse sagt, dass wie die Quantität, so auch die Qualität des Salzes vom gewissen, für jedes Ferment specifischen, Einfluss ist.

Was die Alkaloide anbetrifft, so wird bei Diastas e die Gährung durch Chininum aceticum und Strychninum purum gehemmt, durch Curare, Morphium acet. und Veratrinum sulfuricum beshleunigt. Coffeinum purum bleibt ohne Einfluss. Bei dem Speichelferment hemmt am stärksten Veratrinum sulf. Coffeinum ist wieder wirkungslos, alle anderen besehleunigen, Curare abermals am stärksten. - Darans sehen wir, dass Alkaloide ebenfalls specifisch auf verschiedene Fermente wirken. Bei Pankreasferment sah Nasse durchweg bei allen Alkaloiden eine Beschleunigung. 
Ich habe die Literatur vielleicht zu weitläufig behandelt, dies aber in der Absicht, nm dem geneigten Leser womöglich ein Ganzes iiber die zu behandelnde Frage vorzustellen; die Literatur ist, wie man sieht, nicht gross. Ehe ich meine Experimente beschreibe, sei mir gêstattet, einige Worte über den

\section{Plan der Versuche}

zu sagen. Um den Einfluss der Salze und Alkaloide auf die. Magenfermentation zu untersuchen, wählte ich folgende 10 Salze: $\mathrm{NaCl}, \mathrm{Na}_{2} \mathrm{SO}_{4}$ (mit und ohne Krystallwasser), $\mathrm{NaNO}_{3}, \mathrm{KCl}$, $\mathrm{KNO}_{3}, \mathrm{~K}_{2} \mathrm{SO}_{4}, \mathrm{NH}_{4} \mathrm{Cl},\left(\mathrm{NH}_{4}\right)_{2} \mathrm{SO}_{4}, \mathrm{NH}_{4} \mathrm{NO}_{3}$ und $\left.\mathrm{Na}_{2} \mathrm{~B}_{4} \mathrm{O}_{7}{ }^{1}\right)$. Jedes dieser Salze wurde in folgenden sechs Quantitäten: $0,5 \mathrm{gr}, 1,0 \mathrm{gr}$, $2,0 \mathrm{gr}, 4,0 \mathrm{gr}, 6,0 \mathrm{gr}$ und $8,0 \mathrm{gr}$ benutzt. Auf diese Weise erhielt ich mehr denn 60 Resultate behufs der Wirkung der Salze. Alsdann machte ich Versuche mit folgenden 6 Alkaloiden: Morphium muriaticum, Stryehninum purum, Chininum sulfuricum, Veratrinum.purum, Narcotinum und Digitalinum (Merck, Darmstadt). Ein jedes Alkaloid benutzte ich in folgenden drei Quantitäten: 0,1 gran, 0,2 gran und 0,5 gran. Die Folge dieser Versuche waren also 18 Resultate.

Damit beendigte ich meine Versuche, an denen die Zeit mir knapp zugemessen war, die Concurrenzschrift musste nämlich zum Termin abgeliefert werden.

\section{Abschnitt.}

Der Einfluss der Salze auf die Verdaung.

Materialien. Um die Magenfermentation künstlich herzustellen, sind zwei Bestandtheile nothwendig: das Substrat und die Verdaunngsflüssigkeit. Als erstes benutzte ich den wohl gereinigten Blutfaserstoff. Die in demselben sich befindende Wassermenge wurde vorher durch Austrocknen des Fibrins bestimmt, so

1) Dumas (in der Sitzung der Akademie der Wissenschaften vom 5. Angust 1872) hat zuerst auf die schädliche Einwirkung des $\mathrm{Na}_{2} \mathrm{~B}_{4} \mathrm{O}_{7}$ auf diastatische Fermente, wie: Emulsin, Diastase, Mirosin etc. aufmerksam gemacht. Durch Borax wird die Wirkung dieser Fermente auf Stärke, Amygdalin u. s. w. bei $70^{\circ} \mathrm{C}$, aufgehoben (Gazette medicale XLIII. 1872. Nr. 35, p. 423 und Berichte der deutschen chem. Gesellschaft V, p. 826). 
dass alle Resultate dieser Arbeit auf wasserfreies Fibrin berechnet worden sind. Um die Verdauungsflisssigkeit herzustellen, nahm ich die vom Pylorustheile lospräparirte Magenschleimhaut eines Rindes, und nachdem ich sie in feine Stiickehen zerschnitten habe, goss ich darauf reines Glycerin im Verhältniss $1: 1 \mathrm{~d}$. i. gleiche Theile Glycerin und Schleimhaut. Nach 5-6 Tagen konnte das Infus schon benutzt werden. Alsdann goss ich auf $100 \mathrm{ccm}$ destillirten Wassers 0,5 Salzsäure (spec. Gew. 1,2 ${ }^{1}$ ), dieser Lösung gab ich $7 \mathrm{ccm}$ des Glycerininfuses zu. (Diese Quantität war genügend, um eine Verdauung des Fibrins zu bewirken, was durch einige Probeversuche bestätigt wurde.)

Methode. Auf eine gewisse Quantität Fibrins (gewöhnlich $1,0 \mathrm{gr}$ ), dessen Wasserinhalt mir bekannt war, wurden $100 \mathrm{ccm}$ obiger Verdauungsflüssigkeit gegossen. Ein mit diesen Bestandtheilen gefülltes Glasgefäss, von der Form eines Ballons, wurde in ein Wasserbad von $40-45^{\circ} \mathrm{C}$. gestellt; diese Temperatur wurde mittelst einer kleinen Flamme beständig auf dieser Höhe gehalten. Das Gefäss blieb im Bade 24, zuweilen auch 48 Stunden. Dann wurde die ganze Fliissigkeit durch gewogenes, sehr trockenes schwedisches Papier filtrirt, das auf dem Filter bleibende unverdaute Fibrin einige Male mit heissem Wasser gewaschen, dann sammt dem Filter so lange bei $100^{\circ} \mathrm{C}$. getrocknet, bis es in zwei nach einander folgenden Wägungen keinen Unterschied mehr zeigte, dann das Gewicht des Filtrirpapiers von dem jetzt gefundenen abziehend, erfuhr ich das Gewicht des unverdaut gebliebenen Fibrins. In ein und dasselbe Wasserbad stellte ich gewöhnlich 2-4. Ballons; einen von denselben liess ich ohne Salz; er diente, als Norm der Verdaung; zum Vergleiche mit den anderen, denen verschiedene Quantitäten verschiedener Salze resp. Alkaloide zugegeben wurden. Einem Spezialisten wird diese Arbeitsbeschreibung vielleicht allzu weitläufig erscheinen; ich fühlte mich jedoch gezwungen, sie mitzutheilen, aus Ruicksicht auf die Beschwerden, die ich beim Beginn meiner Versuche, einer guten Technik-Beschreibung ermangelnd, erfahren habe; ich musste den Vorbereitungen mehr Zeit widmen, als den Versuchen selbst. Einen andern Forscher vor diesen Schwierigkeiten zu schonen war der

1) Die Säure von diesem spez. Gewicht enthält $0,228 \%$ reinen ChlorWasserstoffs. 
Ueber den Einfluss einiger Salze und Alkaloiden auf die Verdauung. 297 Zweck obiger Zeilen. Jetzt will ich den ersten Versuch minutiös beschreiben, in den folgenden aber werde ich nur die Zahlenresultate anführen, um den Leser mit Wiederholungen nicht zu langweilen.

\section{Versuch. NaCl.}

$\mathrm{Zu}$ zwei gleichen Theilen Faserstoffs à $2 \mathrm{gr}$ d. i. à $1,828 \mathrm{gr}$ wasserfreien Faserstoffs erhaltend, wurden zu je einem Theile $200 \mathrm{cem}$ der Verdauungsflüssigkeit gesetzt. Dem ersten Ballon (I) wurde $0,5 \mathrm{gr}$ wasserfreies $\mathrm{NaCl}$ beigesetzt, der zweite Theil blieb ohne Salz. Nachdem die Ballons 45 Stunden im Wasserbade gestanden, bekam ich auf oben beschriebene Weise (s. Methode) folgende Resultate:

I. Faserstoff genommen 1,828. Geblieben auf dem Filter 0,116. Verdaut 1,702.

II. Faserstoff 1,828. Geblieben 0,169. Verdant 1,659 .

Tabelle I.

$\mathrm{NaCl}$.

\begin{tabular}{|c|c|c|c|c|c|c|c|}
\hline & $\mathrm{NaCl}$. & Zeit. & 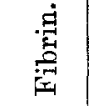 & $\begin{array}{c}\text { Flüssig- } \\
\text { keit. }\end{array}$ & $\begin{array}{c}\text { Ge- } \\
\text { blieben. }\end{array}$ & Verdaut. & Bemerkung. \\
\hline $\begin{array}{r}\text { I } \\
\text { II }\end{array}$ & 0,5 & $\begin{array}{l}45 \mathrm{~S} . \\
45\end{array}$ & $\begin{array}{l}1,828 \\
1,828\end{array}$ & $\begin{array}{l}200 \\
200\end{array}$ & $\begin{array}{l}0,116 \\
0,169\end{array}$ & $\begin{array}{l}1,702 \\
1,659\end{array}$ & $2,6 \%$ Beschleunigung $\left.{ }^{1}\right)$. \\
\hline
\end{tabular}

Bei einer Quantität von $0,5 \mathrm{gr} \mathrm{NaCl}$ sehen wir 2,6\% Beschleunigung der Verdauug; nun lasse ich eine Gesammttabelle, die NaCl-Wirkung beweisend, folgen; alle Versuche wurden auf die eben beschriebene Weise angestellt.

1) Berechnet aus der Proportion 1,702:1,659=x:100

$$
\begin{aligned}
x & =102,6 \\
\text { Beschl } & =102,6-100=2,6 .
\end{aligned}
$$


Louis Wolberg:

Tabelle II.

$\mathrm{NaCl}$.

\begin{tabular}{|c|c|c|c|c|c|c|c|}
\hline & $\mathrm{NaCl}$. & Zeit. & $\begin{array}{l}\text { Flüssig- } \\
\text { keit. }\end{array}$ & 范 & $\begin{array}{c}\mathrm{Ge}^{-} \\
\text {blieben. }\end{array}$ & Verdaut & Einfluss. \\
\hline I & 0,5 & $45 \mathrm{~S}$. & 200 & 1,828 & 0,116 & 1.702 & Beschleunigung $2,6 \mathrm{pCt}$. \\
\hline II & 1,0 & 21 & 100 & 0,907 & 0,055 & 0,852 & Hemmang 2,3 pCt. \\
\hline II & 2,0 & 21 & 100 & 0.907 & 0,054 & 0,853 & Hem. 2,3 pCt. \\
\hline 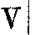 & 4,0 & 21 & 100 & 0,829 & 0,073 & 0,756 & Hem. 4,0 \\
\hline V & 6,0 & 21 & 100 & 0,829 & 0,082 & 0,747 & Hem. 5,4 \\
\hline VI & 8,0 & 21 & 100 & 0,829 & 0,478 & 0,351 & Hem. 44,6" \\
\hline
\end{tabular}

Wenn ich die Zahlen-Resultate mit Worten ausdricke, so sehen wir, dass $\mathrm{NaCl}$ (wasserfrei) in kleinen Mengen die Verdauung ein wenig befördert hat, aber schon die Menge eines Gramms war genïgend, um die Verdauung zu hemmen; diese Hemmang wuchs mit der Vergrösserung des Salzquantums, jedoch hielt sie mit derselben durchaus nicht gleichen Schritt, so z. B. bei $6,0 \mathrm{gr}$ ist die Hemmung nur $5,4 \%$, bei $8,0 \mathrm{gr}$ hingegen $44.6 \%$.

Mit $\mathrm{Na}_{2} \mathrm{SO}_{4}$ veranstaltete ich zwei Reihen von Versuchen, mit wasserfreien $\mathrm{Na}_{2} \mathrm{SO}_{4}$ und mit dem Krystall-Wasser noch enthaltendem Salze; die Resultate und besonders die Verschiedenheiten in der Wirkung beider waren staunenswerth:

Tabelle III.

Wasserfreies $\mathrm{Na}_{2} \mathrm{SO}_{4}$.

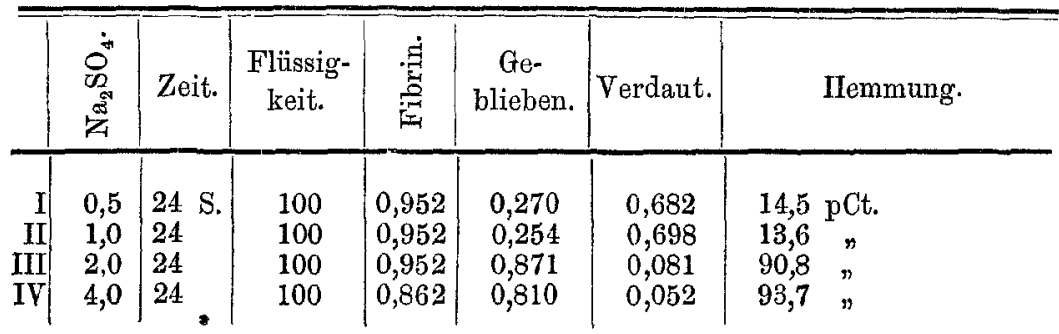

Tabelle IV.

Krystallisches $\mathrm{Na}_{2} \mathrm{SO}_{4}$ (mit $10 \mathrm{H}_{2} \mathrm{O}$ ).

\begin{tabular}{|c|c|c|c|c|c|c|c|}
\hline & 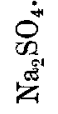 & Zeit. & $\begin{array}{c}\text { Flüssig- } \\
\text { keit. }\end{array}$ & 葛 & $\begin{array}{c}\text { Ge- } \\
\text { blieben. }\end{array}$ & Verdaut. & Hemmung. \\
\hline II & $\begin{array}{l}1,0 \\
2,0\end{array}$ & $\begin{array}{l}24 \mathrm{~S} . \\
24\end{array}$ & $\begin{array}{l}100 \\
100\end{array}$ & $\begin{array}{l}0,862 \\
0,862\end{array}$ & $\begin{array}{l}0,043 \\
0,057\end{array}$ & $\begin{array}{l}0,819 \\
0,805\end{array}$ & $\begin{array}{l}2,2 \text { pCt. } \\
4,0 \%\end{array}$ \\
\hline
\end{tabular}


Ueber den Einfluss einiger Salze und Alkaloiden auf die Verdauung. 299

Also 0,5 gr amorphes $\mathrm{Na}_{2} \mathrm{SO}_{4}$ hemmt die Verdaung um $14,5 \%$, hingegen 1,0 gr krystallisehes nur um 2,2\%. Zwei Gramm wasserfreien $\mathrm{Na}_{2} \mathrm{SO}_{4}$ hemmen um 90,8\%, zwei Gramm krystalli sches nur um $4 \%$. Aehnliche Resultate, deren Glaubwürdigkeit ich durch einen Control-Versuch bestätigt habe, erhielten Ebstein und Müller (1. e.) und sprachen daher die Meinung aus, dass die Salze auf Fermente wirken, indem sie dieselben des zur Fermentation unentbehrlichen Wassers beranben; die wasserfreien Salze sind mehr hygroskopiseh, als Krystallwasser haltende und hemmen deswegen die Fermentation viel stärker. Sie sehen, dass meine Versuche glänzend zur Illustration und Nachweis dieser Theorie dienen können.

Tabelle V.

$\mathrm{NaNO}_{3}$.

\begin{tabular}{|c|c|c|c|c|c|c|c|}
\hline & $\frac{\mathscr{D}^{\circ}}{\overbrace{}^{\circ}}$ & Zeit. & $\begin{array}{c}\text { Flüssig- } \\
\text { keit. }\end{array}$ & $\begin{array}{l}\text { 密 } \\
\text { 窟 }\end{array}$ & $\begin{array}{c}\text { Ge- } \\
\text { blieben. }\end{array}$ & Verdant. & Hemmung. \\
\hline I & 0,5 & $24 \mathrm{~S}$. & 100 & 0,862 & 0,035 & 0,827 & $1,2 \mathrm{pCt}$ \\
\hline $\mathrm{IJ}$ & 1,0 & 24 & 100 & 0,862 & 0,035 & 0,827 & 1,2 \\
\hline III & 2,0 & 24 & 100 & 0,862 & 0,038 & 0,824 & 1,7 \\
\hline IV & 4,0 & 24 & 100 & 0,862 & 0,060 & 0,802 & 3,4 \\
\hline V & 6,0 & 24 & 100 & 0,862 & 0,145 & 0,717 & 13,6 \\
\hline $\mathrm{VI}$ & 8,0 & 24 & 100 & 0,862 & 0,595 & 0,267 & 69,7 \\
\hline
\end{tabular}

$\mathrm{Na} \mathrm{NO}_{3}$ hemmt in allen von mir benutzten Quantitäten. Die Hemmung ist gering bei kleinen Mengen, sie wächst bei $4,0 \mathrm{grm}$, wird beträchtlich bei $6 \mathrm{grm}$, um bei $8 \mathrm{grm}$ circa $70 \%$ zu betragen.

Wenn ich die für die drei Natriumsalze erhaltenen Resultate miteinander vergleiche, so ziehe ich den Schluss, dass amorphes $\mathrm{Na}_{2} \mathrm{SO}_{4}$ am stärksten hemmt, ihm folgt $\mathrm{NaNO}_{3}$, und dann $\mathrm{NaCl}$. Das letzte Salz hat bei kleinen Quantitäten eine verdauungsfördernde Wirkung bewiesen, was ich bei anderen Natriumsalzen nicht beobachtete.

Die durchschnittlicheHemmungsziffer wird für $\mathrm{Na}_{2} \mathrm{SO}_{4}-53,1 \%$, für $\mathrm{NaNO}_{3}-15,1 \%$ und $\mathrm{NaCl}-11,7 \%$ betragen.

Jetzt wenden wir uns zu den Kalisalzen, und beginnen mit 
Tabelle VI.

$\mathrm{KCl}$.

\begin{tabular}{|c|c|c|c|c|c|c|}
\hline $\mathrm{KCl}$ & Zeit. & $\begin{array}{l}\text { Flüssig- } \\
\text { keit. }\end{array}$ & $\begin{array}{l}\text { 莒 } \\
\text { 窧 }\end{array}$ & $\begin{array}{c}\mathrm{Ge}- \\
\text { blieben. }\end{array}$ & Verdant. & Bemerkungen. \\
\hline \begin{tabular}{l|l}
$\mathbf{I}$ & 0,5 \\
$1, \mathrm{I}$ & 1,0 \\
$\mathbf{V}$ & 2,0 \\
$\mathbf{V}$ & 4,0 \\
& 8,0
\end{tabular} & $\begin{array}{l}24 \mathrm{~S} . \\
24 \\
24 \\
24 \\
24\end{array}$ & $\begin{array}{l}100 \\
100 \\
100 \\
100 \\
100\end{array}$ & $\begin{array}{l}0,985 \\
0,985 \\
0,985 \\
0,985 \\
0,985\end{array}$ & $\begin{array}{l}0,052 \\
0,040 \\
0,035 \\
0,082 \\
0,871\end{array}$ & $\begin{array}{l}0,933 \\
0,945 \\
0,950 \\
0,903 \\
0,114\end{array}$ & $\begin{array}{l}\text { Hem. } 1,5 \text { pCt. } \\
\text { Hem. 0,3 " } \\
\text { Beschl. 0,4 pCt. } \\
\text { Hem. 5,2 pCt. } \\
\text { Hem. } 89,0 \text { pCt. }\end{array}$ \\
\hline
\end{tabular}

Dieses Salz ist in seiner Wirkung so unbeständig, wie ein Herbstwetter. In der kleinsten Quantität $(0,5 \mathrm{grm})$ hemmt es, die Hemmung wird aber bei 1 grm geringer, um bei $2 \mathrm{grm}$ sogar in Beschleunigung iiberzugehen, dann haben wir bei 4 und 8 grm wieder eine Hemmung, und zwar $89 \%$ bei der letzt gebrauchten Menge.

Tabelle VII.

$\mathrm{KNO}_{3}$.

\begin{tabular}{|c|c|c|c|c|c|c|c|}
\hline & $\mathrm{KNO}_{3}$ & Zeit. & $\begin{array}{c}\text { Flüssig- } \\
\text { keit. }\end{array}$ & 运 & $\begin{array}{c}\text { Ge- } \\
\text { blieben. }\end{array}$ & Verdaut. & Hemmung. \\
\hline I & 0,5 & $24 \mathrm{~S}$. & 100 & 0,985 & 0,044 & 0,941 & $1,0 \mathrm{pCt}$. \\
\hline II & 1,0 & & 100 & 0,985 & 0,056 & 0,929 & 2,2 \\
\hline III & 2,0 & 24 & 100 & 0,985 & 0,059 & 0,926 & 2,5 \\
\hline IV & 4,0 & 24 & 100 & 0,887 & 0,064 & 0,823 & 3,4 \\
\hline $\mathrm{V}$ & 6,0 & 24 & 100 & 0,887 & 0,093 & 0,794 & 6,8 \\
\hline VI & 8,0 & 24 & 100 & 0,887 & 0,452 & 0,435 & 49,0 \\
\hline
\end{tabular}

Bei allen benutzten Quantitäten sehen wir Hemmung, die jedoch, bei $8 \mathrm{grm}$ ausgenommen, unbedeutend ist. In dieser Hinsicht gleicht $\mathrm{KNO}_{3}$ dem $\mathrm{NaNO}_{3}$, das die Verdauung ebenfalls schwach gehemmt hat. Bei $8 \mathrm{grm} \mathrm{KNO}_{3}$ ist die Hemmung grösser als bei $8 \mathrm{grm} \mathrm{NaNO}_{3}$. Mit $\mathrm{KCl}$ verglichen, wirkt $\mathrm{KNO}_{3}$ viel beständiger, aber $\mathrm{KCl}$ hemmt bei $8 \mathrm{grm}$ fast zwei Mal stärker als $\mathrm{KNO}^{3}$. 
Ueber den Einfluss einiger Salze und Alkaloiden auf die Verdaung. 301

Tabelle VIII.

$\mathrm{K}_{2} \mathrm{SO}_{4}$.

\begin{tabular}{|c|c|c|c|c|c|c|c|}
\hline & $\overbrace{}^{*}$ & Zeit. & $\begin{array}{c}\text { Flüssig" } \\
\text { keit. }\end{array}$ & 量 & $\begin{array}{c}\text { Ge- } \\
\text { blieben. }\end{array}$ & Verdaut. & Bemerkungen. \\
\hline I & 0,5 & $24 \mathrm{~S}$. & 100 & 0,887 & 0,046 & 0,841 & Beschl, 0,5 pCt. \\
\hline II & 1,0 & 24 & 100 & 0,887 & 0,064 & 0,823 & Hem. 2 pCt. \\
\hline III & 2,0 & 24 & 100 & 0,887 & 0,125 & 0,762 & Hem. $9^{\circ}$ \\
\hline IV & 4,0 & 24 & 100 & 0.905 & 0,153 & 0,752 & Hem. $14 "$ \\
\hline $\mathrm{V}$ & 6,0 & 24 & 100 & 0,905 & 0,211 & 0,694 & Hem. 20,6 pCt. \\
\hline VI & 8,0 & 24 & 100 & 0,905 & 0,282 & 0,623 & Hem. 28,8 \\
\hline
\end{tabular}

Auch dieses Salz wirkt hemmend; allerdings bei kleinen Mengen sehen wir eine minimale Beschlennigung; die geht aber schon bei $1 \mathrm{grm}$ in Hemmung tiber, wächst progressiv bei grösseren Mengen, erreicht jedoch, sogar bei $8 \mathrm{grm}$, nicht mehr als $28 \%$.

Die drei Kalisalze vergleichend, finden wir die grösste Hemmung bei $\mathrm{KCl}$, dessen Durchschnittszahl $24 \%$ gleicht, ihm folgen $\mathrm{K}_{2} \mathrm{SO}_{4}$ mit $14,8 \%$ und $\mathrm{KNO}_{3}$ mit $10,8 \%$ Hemmung. Bei Natrium-Verbindungen hemmte $\mathrm{Na}_{2} \mathrm{SO}_{4}$ am stärksten, dann kamen $\mathrm{NaNO}_{3}$ und $\mathrm{NaCl}$. Der Untersehied in der Wirkung der Kaliand Natronsalze wird besonders bei Vergleich der schwefelsauren Verbindungen ersichtlich, so z. B. hemmt $\mathrm{Na}_{2} \mathrm{SO}_{4}$ bei $4 \mathrm{grm}$ um $93,7 \%, \mathrm{~K}_{2} \mathrm{SO}_{4}$ hingegen bei $8 \mathrm{grm}$ nur um $28,0 \%$, folglich drei Mal schwächer bei einer zwei Mal grösseren Quantität.

Sehr nengierig war ich die Wirkung der amoniakalischen Salze beobachten zu können; es schien mir a priori, auf Nasse's Versuche gestützt, dass diese Salze die Fermentation resp. Verdauung beschleunigen müssen; Nasse (1. c.) sah die Zuckerinversion immer durch Ammon-Salze beschleunigt. Derselbe Forscher sagt weiter unten in seiner Arbeit (1. c. siehe Literatur), dass dieselben Salze nicht analog wirken auf yersehiedene Fermente; durch ein und dasselbe Salz wird die Wirkung eines Ferments gehemmt, eines anderen beschleunigt. Einen schlagenden Beweis liefern fitr diesen Satz folgende von mir angestellte Versuche: 
Tabelle IX.

$\mathrm{NH}_{4} \mathrm{Cl}$.

\begin{tabular}{|c|c|c|c|c|c|c|c|}
\hline & 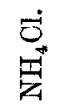 & Zeit. & $\begin{array}{c}\text { Flüssig- } \\
\text { keit. }\end{array}$ & 蒫 & $\begin{array}{c}\text { Ge- } \\
\text { blieben. }\end{array}$ & Verdaut. & Bemerkungen. \\
\hline $\mathrm{I}$ & 0,5 & $24 \mathrm{~S}$. & 100 & 0,905 & 0,053 & 0,852 & Hem. 1,4 pCt. \\
\hline III & 1,0 & & 100 & 0,905 & 0,073 & 0,832 & Hem. 3,7 \\
\hline III & 2,0 & 24 & 100 & 0,905 & 0,068 & 0,837 & Hem. $3,4 "$ \\
\hline $\mathrm{IV}$ & $\overrightarrow{4}, 0$ & 24 & 100 & 0,910 & 0,012 & 0,898 & Beschl. 0,4 "pCt. \\
\hline$\nabla$ & 6,0 & 24 & 100 & 0,910 & 0,025 & 0,885 & Hem. 0,9 pCt. \\
\hline $\mathrm{VI}$ & 8,0 & 24 & 100 & 0,910 & 0,040 & 0,870 & Hem. 2,6 \\
\hline
\end{tabular}

Die bei $0,5-1,0-2,0$ grm geringe hemmende Wirkung dieses Salzes geht bei 4 grm sogar in Beschleunigung iiber, um dann bei 6 und $8 \mathrm{grm}$ wieder zur Hemmung zurïckzukehren. Im Ganzen wird die Verdaung durch dieses Salz wenig beeinflusst, jedenfalls aber ist die Wirkung eine hemmende.

Tabelle $\mathrm{X}$.

$\left(\mathrm{NH}_{4}\right)_{2} \mathrm{SO}_{4}$.

\begin{tabular}{|c|c|c|c|c|c|c|c|}
\hline & $\left(\mathrm{NH}_{4}\right)_{2} \mathrm{SO}_{4}$. & Zeit. & $\begin{array}{c}\text { Flüssig- } \\
\text { keit. }\end{array}$ & 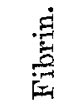 & $\begin{array}{c}\mathrm{Ge}^{-} \\
\text {blieben. }\end{array}$ & Verdaut. & Bemerkungen. \\
\hline I & 0,5 & $24 \mathrm{~S}$. & 100 & 0,910 & 0,104 & 0,806 & Beschl. 0,6 pCt. \\
\hline II & 1,0 & 24 & 100 & 0,910 & 0,178 & 0,732 & Hem. 4,8 pCt. \\
\hline III & 2,0 & 24 & 100 & 0.910 & 0,187 & 0,723 & Hem. $6,0^{\circ}$ \\
\hline IV & 4,0 & 24 & 100 & 0,894 & 0,117 & 0,777 & Hem. 7,8 \\
\hline $\mathbf{V}$ & 6,0 & 24 & 100 & 0,894 & 0,256 & 0,638 & Hem. 24,3 \\
\hline VI & 8,0 & 24 & 100 & 0,894 & 0,318 & 0,576 & Hem. 31,6 \\
\hline
\end{tabular}

Auch dieses Salz hemmt die Verdauung, nur bei $0,5 \mathrm{grm}$ wird dieselbe ein wenig befördert. Die hemmende Wirkung dieser Verbindung im Vergleich mit $\mathrm{NH}_{4} \mathrm{Cl}$ kann man geradezu kolossal nennen, bei $8 \mathrm{grm}$ haben wir $31,6 \%$ Hemmung, eine sogar das $\mathrm{K}_{2} \mathrm{SO}_{4}$ übertreffende hemmende Wirkung.

Tabelle XI.

$\mathrm{NH}_{4} \mathrm{NO}_{3}$.

\begin{tabular}{|c|c|c|c|c|c|c|c|}
\hline & $\dot{\beta}^{\dot{\infty}}$ & Zeit. & $\begin{array}{c}\text { Flüssig- } \\
\text { keit. }\end{array}$ & 吾 & $\begin{array}{c}\text { Ge- } \\
\text { blieben. }\end{array}$ & Verdaut. & Hemmung. \\
\hline $\mathrm{I}$ & 0,5 & $24 \mathrm{~S}$. & 100 & 0,894 & 0,054 & 0,840 & 1,1 pCt. \\
\hline II & 1,0 & & 100 & 0,894 & 0,072 & 0,822 & 3,2 \\
\hline III & 2,0 & 24 & 100 & 0,894 & 0,089 & 0,805 & 5,2 \\
\hline IV & 4,0 & 24 & 100 & 0,900 & 0,057 & 0,843 & $1,8 \%$ \\
\hline V & 6,0 & 24 & 100 & 0,900 & 0,101 & 0,799 & 6,9 \\
\hline VI & 8,0 & 24 & 100 & 0,900 & 0,106 & 0,794 & 7,5 \\
\hline
\end{tabular}


Ueber den Einfluss einiger Salze und Alkaloiden auf die Verdauung. 303

Die hemmende Wirkung dieses Salzes ist in allen benutzten Quantitäten ersichtlich, obgleich nicht sehr beträchtlich; die grösste Hemmung (bei $8 \mathrm{grm}$ ) ist nur 7,5\%.

Die drei amoniakalischen Salze vergleichend, komme ich zur Ueberzengung, dass die Verdaung dureh alle diese Salze gehemmt wird (andere Fermentationen werden durch sie gefördert). Im Vergleiche mit anderen Salzen wirken die amoniakalischen schwach; am stärksten ist $\left(\mathrm{NH}_{4}\right)_{2} \mathrm{SO}_{4}=14,4 \%$, dann $\mathrm{NH}_{4} \mathrm{NO}_{3}=4,3 \%$ und $\mathrm{NH}_{4} \mathrm{Cl}=2,0 \%$.

Tabelle XII.

$\mathrm{Na}_{2} \mathrm{~B}_{4} \mathrm{O}_{7}$ (Borax, Sel de Conserve).

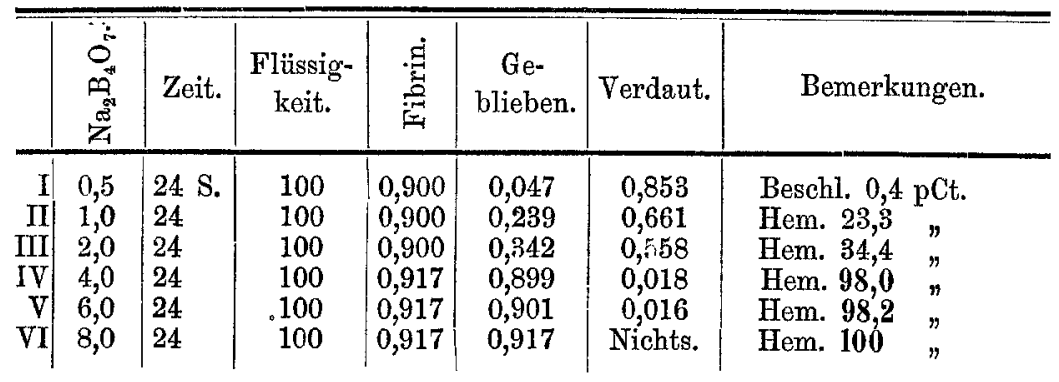

Dieses Salz beschleunigt bei 0,5 grm die Verdaunng, aber von 1 grm beginnend hemmt es dieselbe und zwar sehr beträchtlich, die Hemmung wächst bei grösseren Quantitäten und erreicht bei $8 \mathrm{grm} 100 \%$, d. h. kein einziges Milligramm Faserstoff wurde nach 24 Standen bei dieser Quantität verdaut. In der letzten Zeit wurde die Aufmerksamkeit der Wissenschaft durch einen Brief Prof. E. Cyon's an den Herrn Vorsitzenden des medicinischen Rathes in Petersburg (Dr. Pelikan) auf die desinficirende Wirkung des Sel de Conserve gelenkt; dieses Salz soll ein starkes Desinficiens sein und wird zum inneren Gebrauche während Typhus, Pest und anderen Epidemicen von Cyon empfohlen. 
Die folgende Tabelle soll zur schnellen und vergleichenden Uebersicht der hemmenden Salzewirkung dienen. Indem ich Verbindungen, die verschiedene Säuren enthielten, mit einander verglichen habe, habe ich einige Winke über die Wirkung: der Säuren auf die Verdauung erhalten; diese sollten aber noch selbständig untersucht werden, um bestimmend zu sein:

Tabelle XIII.

Vergleichende Tabelle.

\begin{tabular}{|c|c|c|c|}
\hline Salz. & $\begin{array}{l}\text { Quan- } \\
\text { tität. }\end{array}$ & Einfluss. & Resultate. \\
\hline $\begin{array}{l}\mathrm{NaCl} \\
\mathrm{Na}_{2} \mathrm{SO}_{4} \text { (geschmolzen). } \\
\mathrm{Na}_{2} \mathrm{SO}_{4} \text { (crystal.) } \\
\mathrm{NaNO}_{3} \\
\mathrm{KCl} \\
\mathrm{KNO}_{3} \\
\mathrm{~K}_{3} \mathrm{SO}_{4} \\
\mathrm{NH}_{4} \mathrm{Cl} \\
\mathrm{NH}_{4} \mathrm{NO}_{3} \\
\left(\mathrm{NH}_{4}\right)_{2} \mathrm{SO}_{4} \\
\mathrm{Na}_{2} \mathrm{~B}_{4} \mathrm{O}_{7}\end{array}$ & $\begin{array}{l}0,5 \mathrm{gr} \\
- \\
- \\
- \\
= \\
- \\
-\end{array}$ & 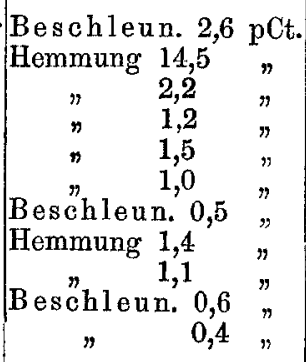 & $\begin{array}{l}\text { Wir sehen, dass 1) amor- } \\
\text { phes } \mathrm{Na}_{2} \mathrm{SO}_{4} \text { am stärksten } \\
\text { hemmt. 2) } \mathrm{NaCl}_{1}\left(\mathrm{NH}_{4}\right)_{2} \mathrm{SO}_{4} \text {, } \\
\mathrm{NH}_{4} \mathrm{Cl} \text { und } \mathrm{Na}_{2} \mathrm{~B}_{4} \mathrm{O}_{7} \text { be- } \\
\text { scleunigen. 3) Säuren } \\
\text { iuben keine besondere Wir- } \\
\text { kung auf die Verdaung } \\
\text { aus; am stärksten hemmt } \\
\mathrm{HCl} \text {, dann } \mathrm{H}_{2} \mathrm{SO}_{4} \text { und } \mathrm{HNO}_{3} \text {. }\end{array}$ \\
\hline $\begin{array}{l}\mathrm{NaCl} \\
\mathrm{Na}_{2} \mathrm{SO}_{4} \text { (geschm.) } \\
\mathrm{Na}_{2} \mathrm{SO}_{4} \text { (crystal.) } \\
\mathrm{NaNO}_{3} \\
\mathrm{KCl} \\
\mathrm{K}_{2} \mathrm{SO}_{4} \\
\mathrm{KNO}_{3} \\
\mathrm{NH}_{4} \mathrm{Cl} \\
\left(\mathrm{NH}_{4}\right)_{2} \mathrm{SO}_{4} \\
\mathrm{NH}_{4} \mathrm{NO}_{3} \\
\mathrm{Na}_{2} \mathrm{~B}_{4} \mathrm{O}_{7}\end{array}$ & $\begin{array}{l}1,0 \mathrm{gr} \\
- \\
= \\
- \\
- \\
= \\
= \\
-\end{array}$ & $\begin{array}{cll}\text { Hemmung } & 2,3 & \text { pCt. } \\
" & 13,6 & \\
" & 4,0 & " \\
" & 1,2 & " \\
" & 0,3 & " \\
" & 2,0 & " \\
" & 2,2 & " \\
" & 3,7 & " \\
" & 4,8 & " \\
" & 3,2 & " \\
. & 23,3 & \end{array}$ & $\begin{array}{l}\text { 1) Alle Salze hemmen } \\
\text { bei dieser Quantität. 2) } \mathrm{Am} \\
\text { stärksten hemmt } \mathrm{Na}_{2} \mathrm{~B}_{4} \mathrm{O}_{7} \text {, } \\
\text { am schwächsten KCl. 3) } \\
\text { Von allen Salzen hemmen } \\
\text { die schwefelsauren am } \\
\text { stärksten. }\end{array}$ \\
\hline $\begin{array}{l}\mathrm{NaCl} \\
\mathrm{Na}_{2} \mathrm{SO}_{4} \text { (geschm.) } \\
\mathrm{NaNO}_{3} \\
\mathrm{KCl} \\
\mathrm{K}_{2} \mathrm{SO}_{4} \\
\mathrm{KNO}_{3} \\
\mathrm{NH}_{4} \mathrm{Cl} \\
\mathrm{NH}_{4} \mathrm{SO}_{2} \mathrm{SO}_{4} \\
\mathrm{NH}_{4} \mathrm{NO}_{3} \\
\mathrm{Na}_{2} \mathrm{~B}_{4} \mathrm{O}_{4}\end{array}$ & $\begin{array}{l}2,0 \mathrm{gr} \\
= \\
= \\
= \\
= \\
=\end{array}$ & 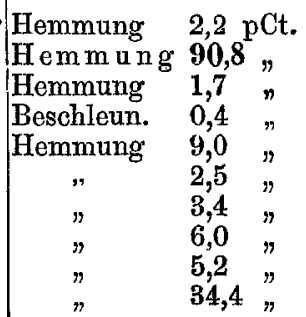 & $\begin{array}{l}\text { 1) Alle Salze, mit Aus- } \\
\text { nahme des } \mathrm{KCl} \text {, hemmen. } \\
\text { 2) } \mathrm{Am} \text { stärksten wirkt } \\
\mathrm{Na}_{2} \mathrm{SO}_{4}, \mathrm{Na}_{2} \mathrm{~B}_{4} \mathrm{O}_{4} \text { und } \\
\mathrm{K}_{2} \mathrm{SO}_{4} \text { 3) Die schwefel- } \\
\text { sauren Salze sind stärker } \\
\text { hemmend, als die salz- } \\
\text { sauren, diese stärker als } \\
\text { die salpetersauren. }\end{array}$ \\
\hline
\end{tabular}


Ueber den Einfluss einiger Salze and Alkaloiden auf die Verdauung. 305

\begin{tabular}{|c|c|c|c|}
\hline Salz. & $\begin{array}{l}\text { Quan- } \\
\text { tität. }\end{array}$ & Einfluss. & Resultate. \\
\hline $\begin{array}{l}\mathrm{NaCl} \\
\mathrm{Na}_{2} \mathrm{SO}_{4} \text { (geschm.) } \\
\mathrm{NaNO}_{3} \\
\mathrm{KCl} \\
\mathrm{K}_{2} \mathrm{SO}_{4} \\
\mathrm{KNO}_{3} \\
\mathrm{NH}_{4} \mathrm{Cl} \\
\left(\mathrm{NH}_{4}\right)_{2} \mathrm{SO}_{4} \\
\mathrm{NH}_{4} \mathrm{NO}_{3} \\
\mathrm{Na}_{2} \mathrm{~B}_{4} \mathrm{O}_{7}\end{array}$ & $\begin{array}{l}4,0 \mathrm{gr} \\
- \\
- \\
- \\
- \\
- \\
-\end{array}$ & 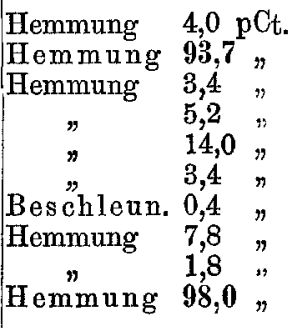 & $\begin{array}{l}\text { 1) AlleSalze, } \mathrm{NH}_{4} \mathrm{Cl} \text { aus- } \\
\text { genommen, hemmen. 2) } \\
\mathrm{Na}_{2} \mathrm{~B}_{4} \mathrm{O}_{7}, \mathrm{Na}_{2} \mathrm{SO}_{4} \text { u. KNO } \\
\text { sind die stärksten. 3) Am } \\
\text { beträchtlichsten hemmen } \\
\text { die schwefelsauren, dann die } \\
\text { salzsauren, dann die sal- } \\
\text { petersauren Verbindungen. }\end{array}$ \\
\hline $\begin{array}{l}\mathrm{NaCl} \\
\mathrm{NaNO}_{3} \\
\mathrm{~K}_{2} \mathrm{SO}_{4} \\
\mathrm{KNO}_{3} \\
\mathrm{NH}_{4} \mathrm{Cl} \\
\left(\mathrm{NH}_{4}\right)_{2} \mathrm{SO}_{4} \\
\mathrm{NH}_{4} \mathrm{NO}_{3} \\
\mathrm{Na}_{2} \mathrm{~B}_{4} \mathrm{O}_{7}\end{array}$ & $\mid \begin{array}{c}6,0 \mathrm{gr} \\
- \\
- \\
- \\
- \\
-\end{array}$ & $\begin{array}{cll}\text { Hemmung } & 5,4 & \text { pCt. } \\
" & 13,6 & \\
" & 14,0 & " \\
" & 6,8 & " \\
" & 0,9 & " \\
" & 24,3 & " \\
" & 6,9 & " \\
" & \mathbf{9 8 , 2} & \end{array}$ & $\begin{array}{l}\text { 1) Alle Salze hemmen. 2) } \\
\text { Am stärksten } \mathrm{Na}_{2} \mathrm{~B}_{4} \mathrm{O}_{7} \\
\left(\mathrm{NH}_{4}\right)_{2} \mathrm{SO}_{4}, \mathrm{~K}_{2} \mathrm{SO}_{4} \mathrm{u} . \mathrm{NaNO}_{3} \text {. } \\
\text { 3) Die schwefelsauren sind } \\
\text { stärker wie andere, die sal- } \\
\text { petersauren aber stärker } \\
\text { wie die salzsauren (vergl. } \\
\text { mit Bemerkg. bei } 4 \mathrm{u} .2 \mathrm{gr} \text { ). }\end{array}$ \\
\hline $\begin{array}{l}\mathrm{NaCl} \\
\mathrm{NaNO} \\
\mathrm{KCl} \\
\mathrm{KNO}_{3} \\
\mathrm{~K}_{2} \mathrm{SO}_{4} \\
\mathrm{NH}_{4} \mathrm{Cl} \\
\mathrm{NH}_{4} \mathrm{SO}_{2} \mathrm{SO}_{4} \\
\mathrm{NH}_{4} \mathrm{NO}_{8} \\
\mathrm{Na}_{2} \mathrm{~B}_{4} \mathrm{O}_{7}\end{array}$ & $\begin{array}{c}8,0 \mathrm{gr} \\
= \\
= \\
= \\
= \\
-\end{array}$ & 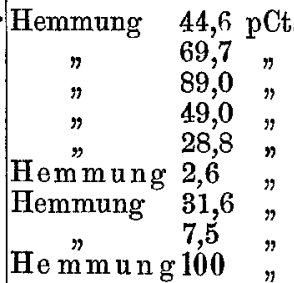 & $\begin{array}{l}\text { 1) Alle Salze hemmen. } \\
2) \text { Am stärksten wirken } \\
\mathrm{Na}_{2} \mathrm{~B}_{4} \mathrm{O}_{7}, \mathrm{KCl}, \mathrm{NaNO}_{3}, \mathrm{NaCl} \text {, } \\
\left(\mathrm{NH}_{4}\right)_{2} \mathrm{SO}_{4}, \mathrm{~K}_{2} \mathrm{SO}_{4}, \mathrm{NH}_{4} \mathrm{NO} \mathrm{O}_{3} \\
\text { und } \mathrm{NH}_{4} \mathrm{Cl} \text { 3) Am stärk- } \\
\text { sten wirken die salpeter- } \\
\text { sauren, dann die schwefel- } \\
\text { sauren und salzsauren Ver- } \\
\text { bindungen. }\end{array}$ \\
\hline
\end{tabular}

\section{Allgemeine Endresultate über die Wirkung der Salze auf die Verdaung.}

Ich untersuchte die Wirkung folgender zehn Salze: $\mathrm{KCl}$, $\mathrm{K}_{2} \mathrm{SO}_{4}, \mathrm{KNO}_{3}, \mathrm{NaCl}, \mathrm{Na}_{2} \mathrm{SO}_{4}, \mathrm{NaNO}_{3}, \mathrm{NH}_{4} \mathrm{Cl}, \mathrm{NH}_{4} \mathrm{NO}_{3},\left(\mathrm{NH}_{4}\right)_{2} \mathrm{SO}_{4}$ und $\mathrm{Na}_{2} \mathrm{~B}_{4} \mathrm{O}_{7}$ und kam zu folgenden Postulaten:

1) Die Verdauung wird durch diese Verbindungen gehemmt.

2) Die Grösse der hemmenden Wirkung hängt nicht nur von der Salzart ab, sondern auch von der Qualität der in der Verbindung sich befindenden Säure.

3) Dem Hemmungsgrade nach kann man diese Salze in folgender Reihe aufstellen: 


$\begin{array}{lc}\mathrm{Na}_{2} \mathrm{SO}_{4} \text { (wasserfrei) } & 59,5 \% \\ \mathrm{Na}_{2} \mathrm{~B}_{4} \mathrm{O}_{7} \text { (wasserfrei) } & 59,3 \\ \mathrm{KCl} & 24,0 \\ \mathrm{NaNO}_{3} & 15,1 \\ \mathrm{~K}_{2} \mathrm{SO}_{4} & 14,8 \\ \left(\mathrm{NH}_{4}\right)_{2} \mathrm{SO}_{4} & 14,4 \\ \mathrm{NaCl} & 11,7 \\ \mathrm{KNO}_{3} & 10,8 \\ \mathrm{NH}_{4} \mathrm{NO}_{3} & 4,3 \\ \mathrm{NH}_{4} \mathrm{Cl} & 2,0\end{array}$

4) Was die Wirkung des $\mathrm{K}, \mathrm{Na}$ und $\mathrm{NH}_{3}$ (auf Säuren nicht geachtet) betrifft, so hemmt $\mathrm{NH}_{3}$ in kleinen Mengen stärker, als die anderen, von 4,0 grm aber angefangen, ist $\mathrm{Na}$ und $\mathrm{K}$ viel stärker, als $\mathrm{NH}_{3}$. In der Wirkung des $\mathrm{Na}$ und $\mathrm{K}$ sah ich keinen besonderen Unterschied.

5) Die mächtigste hemmende Wirkung übt die Sch wefelsäure aus; die Salpetersäure ist der Salzsäure nur um ein Weniges iiberlegen.

6) Nur bei 6 grm sehen wir bei allen Salzen eine hemmende Wirkung. Bei $4 \mathrm{grm}$ beschleunigte $\mathrm{NH}_{4} \mathrm{Cl}$ noch ein wenig.

\section{Abschnitt.}

Der Einfluss der Alkaloide auf die Verdaung.

Materialien und Methode, bei diesen Versuchen von mir gebraucht, waren mit den oben beschriebenen vollkommen identisch. Die Alkaloide benutzte ich in kleinen Mengen von $0,1 \mathrm{Gran}=$ $0,00625 \mathrm{gr} ; 0,2 \mathrm{Gr} .=0,01250$ und $0,5 \mathrm{Gr} .=0,03125 \mathrm{gr}$.

Tabelle XIV.

Morphium muriaticum.

\begin{tabular}{|c|c|c|c|c|c|c|c|}
\hline & $\begin{array}{l}\text { Morphium } \\
\text { muriat. }\end{array}$ & Zeit. & $\begin{array}{c}\text { Flüssig- } \\
\text { keit. }\end{array}$ & 葛 & $\begin{array}{c}\text { Ge- } \\
\text { blieben. }\end{array}$ & Verdaut. & Hemmung. \\
\hline $\begin{array}{l}\text { I } \\
\text { II } \\
\text { III } \\
\text { IV }\end{array}$ & $\begin{array}{c}0,1 \mathrm{gr} \\
0,2 \\
0,5 \\
\text { ohne }\end{array}$ & $\begin{array}{l}24 \mathrm{~S} . \\
24 \\
24 \\
24\end{array}$ & $\begin{array}{l}100 \\
100 \\
100 \\
100\end{array}$ & $\begin{array}{l}0,917 \\
0,917 \\
0,917 \\
0,917\end{array}$ & $\begin{array}{l}0,054 \\
0,055 \\
0,083 \\
0,053\end{array}$ & $\begin{array}{l}0,863 \\
0,862 \\
0,834 \\
0,864\end{array}$ & $\begin{array}{c}0,1 \\
0,3 \text { Ct. } \\
4,0 " \\
-"\end{array}$ \\
\hline
\end{tabular}


Ueber den Einfluss einiger Salze und Alkaloiden auf die Verdauung. 307

Nr. IV liess ich ohne Alkaloid zum Vergleiche mit den anderen. Die Verdauung wird durch Morph. muriat. gehemmt; da die benutzten Mengen klein sind, so ist auch die Hemmung keine grosse, jedoch bei $1 / 2$ Gr. beträgt sie schon $4 \%$.

Tabelle XV.

Strichninum purum.

\begin{tabular}{|c|c|c|c|c|c|c|c|}
\hline & $\begin{array}{l}\text { Strichninum } \\
\text { purum. }\end{array}$ & Zeit. & $\begin{array}{c}\text { Flüssig- } \\
\text { keit. }\end{array}$ & $\frac{\mathscr{E}}{E}$ & $\begin{array}{c}\text { Ge- } \\
\text { blieben. }\end{array}$ & Verdaut. & Einfluss. \\
\hline $\begin{array}{r}\mathrm{I} \\
\mathrm{II} \\
\mathrm{III} \\
\mathrm{IV}\end{array}$ & $\begin{array}{c}0,1 \mathrm{Gr} . \\
0,2 \\
0,5 \\
\text { obne }\end{array}$ & $\begin{array}{l}24 \text { S. } \\
24 \\
24 \\
24\end{array}$ & $\begin{array}{l}100 \\
100 \\
100 \\
100\end{array}$ & $\begin{array}{l}0,906 \\
0,906 \\
0,906 \\
0,906\end{array}$ & $\begin{array}{l}0,020 \\
0,057 \\
0,060 \\
0,037\end{array}$ & $\begin{array}{l}0,886 \\
0,849 \\
0,846 \\
0,869\end{array}$ & $\begin{array}{ccc}\text { Beschl. } & 2,0 & \text { pCt. } \\
\text { Hem. } & 2,3 & \\
" & 2,6 & "\end{array}$ \\
\hline
\end{tabular}

Strichninum hemmt schwächer als Morphium, ja sogar in kleinen Quantitäten beschleunigt es die Verdauung und zwar um $2 \%$.

Tabelle XVI.

Chininum sulfuricum.

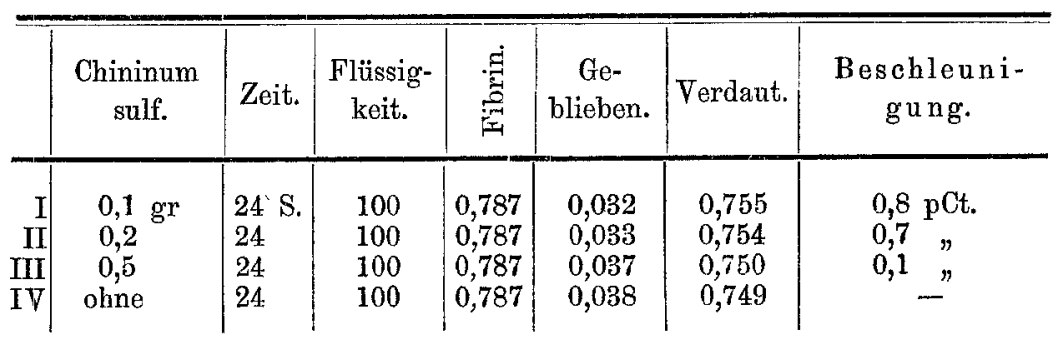

Dieses Alkaloid be schle unigt durchweg die Verdaung; da aber diese Wirkung im verkehrten Verhältnisse zur Vergrösserung der Quantität steht, so ist zu behaupten, dass in noch grösseren Dosen Chinin die Verdauung hemmen würde, was doch so oft am Krankenbett beobachtet wird. 
Tabelle XVII.

Veratrinum purtum.

\begin{tabular}{|c|c|c|c|c|c|c|}
\hline $\begin{array}{l}\text { Veratrinum } \\
\text { pur. }\end{array}$ & Zeit. & $\begin{array}{l}\text { Flüssig- } \\
\text { keit. }\end{array}$ & 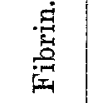 & $\begin{array}{c}\text { Ge- } \\
\text { blieben. }\end{array}$ & Verdaut. & Hemmung. \\
\hline $\begin{array}{l}0,1 \mathrm{Gr} . \\
0,2 \\
0,5 \\
-\end{array}$ & $\begin{array}{l}24 \text { S. } \\
24 \\
24 \\
24\end{array}$ & $\begin{array}{l}100 \\
100 \\
100 \\
100\end{array}$ & $\begin{array}{l}0,787 \\
0,787 \\
0,787 \\
0,787\end{array}$ & $\begin{array}{l}0,040 \\
0,040 \\
0,039 \\
0,036\end{array}$ & $\begin{array}{l}0,747 \\
0,747 \\
0,748 \\
0,751\end{array}$ & \begin{tabular}{cc}
$0,5 \quad \mathrm{pCt}$. \\
$0,5 \quad "$ \\
$0,46 "$ \\
\multicolumn{2}{c}{$"$}
\end{tabular} \\
\hline
\end{tabular}

Einen hemmenden Einfluss iibt Veratrin auf die Verdauung; in allen drei Quantitäten ist diese Hemmung sichtbar, obgleich sehr niedrig.

Tabelle XVIII.

$\mathrm{Narcotinu} \mathrm{m}$.

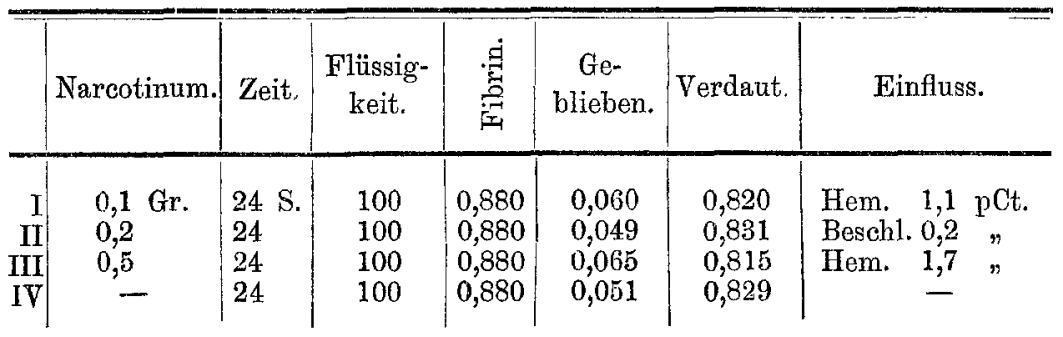

Schwach hemmend ist die Wirkung des Narcotinum; es wirkt schwächer als das zweite von mir untersuchte Alkaloid des Opium's, das Morphium.

Tabelle XIX.

Digitalinum (Merck. Darmstadt).

\begin{tabular}{|c|c|c|c|c|c|c|c|}
\hline & Digitalinum. & Zeit. & $\begin{array}{l}\text { Flüssig- } \\
\text { keit. }\end{array}$ & 离 & $\begin{array}{c}\text { Ge- } \\
\text { blieben. }\end{array}$ & Verdaut. & Hemmung. \\
\hline III & $\begin{array}{l}0,1 \mathrm{Gr} . \\
0,2 \\
0,5 \\
\text { ohne }\end{array}$ & $\begin{array}{l}24 \mathrm{~S} . \\
24 \\
24 \\
24\end{array}$ & $\begin{array}{l}100 \\
100 \\
100 \\
100\end{array}$ & $\begin{array}{l}0,880 \\
0,880 \\
0,880 \\
0,880\end{array}$ & $\begin{array}{l}0,047 \\
0,048 \\
0,051 \\
0,042\end{array}$ & $\begin{array}{l}0,833 \\
0,832 \\
0,829 \\
0,838\end{array}$ & $\begin{array}{c}0,6 \mathrm{pCt.} \\
0,7 " \\
1,5 \quad "\end{array}$ \\
\hline
\end{tabular}

Digitalinum hemmt in allen drei benutzten Quantitäten; die Hemmung wächst mehr oder weniger proportionell mit der Grösse der Quantität. 
Ueber den Einfluss einiger Salze und Alkaloiden auf die Verdauung. 309

Nr. XX.

Vergleichende Tabelle.

\begin{tabular}{|c|c|c|c|}
\hline Alkaloid. & Gran. & Einfluss. & Bemerkungen. \\
\hline $\begin{array}{l}\text { Morph. muriat. } \\
\text { Strichninum } \\
\text { Chinin. sulf. } \\
\text { Veratrinum } \\
\text { Narcotinum } \\
\text { Digitalinum }\end{array}$ & $\begin{array}{l}0,1 \\
- \\
- \\
-\end{array}$ & 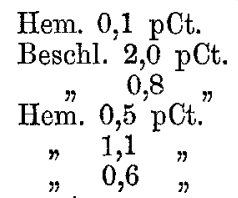 & $\begin{array}{l}\text { Am stärksten hemmt Nar- } \\
\text { cotinum, dann Digitalinum, Ve- } \\
\text { ratrinum und Morphium. Chinin } \\
\text { und Strichnin beschleunigen. }\end{array}$ \\
\hline $\begin{array}{l}\text { Morph. muriat. } \\
\text { Strichninum } \\
\text { Chininum sulf. } \\
\text { Veratrinum } \\
\text { Narcotinum } \\
\text { Digitalinum }\end{array}$ & $\begin{array}{l}0,2 \\
- \\
= \\
-\end{array}$ & $\begin{array}{l}\text { Hem. 0,3 pCt. } \\
2,3 " \\
\text { Beschl. 0,7 } \\
\text { Hem. 0,5 } \\
\text { Beschl. 0,2" } \\
\text { Hem. 0,7 " }\end{array}$ & $\begin{array}{l}\text { Am stärksten hemmt Strichnin, } \\
\text { der bei } 0,1 \mathrm{Gr} \text {. stark beschleu- } \\
\text { nigt. Chininum und Narcotinum } \\
\text { beschleunigen. }\end{array}$ \\
\hline $\begin{array}{l}\text { Morph. mur. } \\
\text { Strichninum } \\
\text { Chin. sulf. } \\
\text { Narcotinum } \\
\text { Veratrinum } \\
\text { Digitalinum }\end{array}$ & $\begin{array}{l}0,5 \\
- \\
- \\
-\end{array}$ & $\begin{array}{l}\text { Hem. } 4,0 \text { pCt. } \\
2,6 " \\
\text { Beschl. } 0,1 " \\
\text { Hem. } 1,7 " \\
\Rightarrow 0,46 " \\
" 1,5 "\end{array}$ & $\begin{array}{l}\text { Am stärksten hemmtMorphium } \\
\text { muriaticum, alsdann Strichninum, } \\
\text { Narcotinum, Digitalinum und } \\
\text { Veratrinum. Nur Chininum } \\
\text { sulfuricum beschleunigt. }\end{array}$ \\
\hline
\end{tabular}

\section{Allgemeine Endresultate über die Wirkung der Alkaloide anf ' die Verdaumng.}

Von den sechs untersuchten Alkaloiden wirken: 1) fünf hemmend auf die Verdauung, und nur Chininum sulf. beschleunigt dieselbe; aber diese Beschleunigung steht im umgekehrten Verhältniss zur Quantität der Alkaloide. 2) Die Hemmungen sind nicht gross, weil die Quantitäten der Alkaloide allzu gering waren. 3) a) Durchschnittszahlen der Hemmung

Morphium $\quad 1,466$

Strichninum $\quad 0,966$

Digitalinum $\quad 0,933$

Narcotinum $\quad 0,866$

Veratrinum $\quad 0,440$ 
b) Durchschnittsaahl der Beschleunigung

Chininum sulfur. $0,533 \%$.

4) Obgleich laut dieser Tabelle Morphium am stärksten hemmt, so ist dies nur für das Quantum von 0,5 Gran zutreffend, hingegen bei 0,1 und 0,2 Gran hemmt es viel schwäeher, als andere Alkaloide.

\section{Ueber Wärmetönung bei Fermentwirkungen.}

\section{Von}

v. Nägeli in München.

In der "Theorie der Gährung " habe ich die Wirkung der (unorganisirten) Fermente und der (organisirten) Hefenpilze mit einander verglichen und im Gegensatze zu den herrschenden Ansichten gezeigt, dass zwischen beiden Processen nicht Uebereinstimmung, sondern gerade in den massgebenden Eigenschaften eine charakteristische Verschiedenheit besteht. Unter den Momenten, welche diese Verschiedenheit bedingen, betrifft eines die Wärmetönung, indem bei dem einzigen Gährprocess, den wir genau kennen, nämlich bei der Alkoholgährung, sicher Wärme frei, bei dem einzigen Process der Fermentwirkung, den wir etwas genauer kennen, nämlich bei der Invertirung des Rohrzuckers, höchst wahrseheinlich Wärme aufgenommen wird.

Gegen diese Theorie hat sich A. Kunkel ${ }^{1}$ ) ausgesprochen. Nach seiner Darlegung würde bei der Invertirung des Rohrzuckers (durch Invertin oder Schwefelsäure) nicht Wärme aufgenommeu, sondern abgegeben, und es würde somit die Wärmetönung bei der Fermentwirkung die nämliche sein wie bei der Gährwirkung ${ }^{2}$ ).

1) Ueber Wärmetönung bei den Fermentationen in Pflüger's Archiv f. Phys. Bd. XX, 509.

2) Bezüglich der Terminologie habe ich in der "Theorie der Gährung" bereits bemerkt, dass ich Fermentwirkung nur als Concession an den jetzt allgemein gewordenen Sprachgebrauch im Gegensatz zu Gähr- oder Hefenwir- 